\title{
An Overview of COVID-19 Vaccine Safety and Post-marketing Surveillance Systems
}

Manal Mohammed Younus, BS Pharm, MS, PhD'; Ali Azeez Al-Jumaili, BS Pharm, MS, MPH, PhD²

${ }^{1}$ Director of Iraqi Pharmacovigilance Center, Ministry of Health, Baghdad, Iraq

${ }^{2}$ University of Baghdad College of Pharmacy, Baghdad, Iraq

\section{Why study COVID-19 vaccine safety}

Building the safety of COVID-19 vaccines is a continuous process throughout the vaccine life cycle; It starts early at the clinical trial in different phases and continues to post-marketing to ensure that the vaccine used are very safe. Before clinical trials, the vaccine's physical, chemical, and biological evaluations are performed. Animal studies can also help to collect preliminary information related to safety and efficacy. The protocols of human clinical trials and, afterward, surveillance systems will identify all short-term safety concerns through risk minimization measures [1]. The objective of this article is to describe COVID-19 vaccine safety and pre-and postmarketing surveillance systems.

Since some candidate vaccines use platform technologies that have not been extensively tested in human subjects to date, such as viral vectors, mRNA, and nanoparticle constructs, they may cause potential adverse reactions due to abnormal immune responses. Thus, pre-and post-licensure, comprehensive safety studies are critical [2].

Clinical trials are conducted before vaccine licensure to assess both safety and efficacy. Randomized clinical trials are the gold standard as they reduce confounding and bias. Strict inclusion and exclusion criteria also reduce risk to study participants. However, such studies have limitations. They cannot evaluate adverse events in persons with specific characteristics excluded from studies or adverse events with the long-delayed onset and uncommon adverse reactions [3]. Therefore, post-marketing safety studies, including surveillance systems, are crucial to collect the missing data, potential risks data and better understand the vaccine safety profile.

\section{Corresponding author:}

Ali Azeez Al-Jumaili, BS Pharm, MS, MPH, PhD

Faculty member, University of Baghdad College of Pharmacy

Baghdad, Iraq

Adjunct faculty, The University of lowa

College of Pharmacy, lowa, USA.

Email: ali.baraak@copharm.uobaghdad.edu.iq
Pre vs. post-marketing of COVID-19 vaccine safety

The traditional vaccine development would need 15 years or longer, starting from the discovery and design, then the preclinical testing that might take 5-10 years. At this time, an investigational new drug (IND) application is submitted, and the vaccine candidate is tested in phases I, II, and III. Phase I clinical trial requires about three years with around 10-100 participants. Another 2-3 years for phase II with 100-1000s participants, and about three years for phase III with 3000 50.000 participants. If the predetermined endpoints are reached at the end of phase III trials, a biologics license application (BLA) is submitted, reviewed by regulatory authorities, and the vaccine is eventually authorized. Largescale development starts after that [1].

The COVID-19 pandemic vaccine development can take accelerated timelines from 10 months to 18 months, where both pre-clinical testing and phase I take around 6-9 months, phases II and III also take 6-9 months before granting the emergency authorization [4]. This is because the discovery process was omitted due to experience gained from the initial development of vaccines for the severe acute respiratory syndrome (SARS-CoV) and the Middle East Respiratory Syndrome Coronavirus (MERS-CoV). Existing procedures were implemented, and phase $\mathrm{I} / \mathrm{II}$ trials began. After the interim review of phase I/II results, phase III trials began, with multiple clinical trial phases run simultaneously. In the meantime, vaccine manufacturers have begun large-scale production [1].

Currently, there are around 320 vaccine candidates, 97 in clinical testing and 17 in use, of whom seven were given emergency Use Authorization (EUA) [5]. All the current COVID19 vaccines were granted a EUA after carefully weighing the benefits and risks of the vaccine, taking into accounts the targeted population, the indication for use, and the safety studies after marketing approval to be continued [6].

Types of safety surveillance systems for vaccines

Monitoring the safety of vaccines is a complex ongoing process. Vaccine safety must be proactive, timely, and conducted with rigor and objectivity. According to the World Health Organization (WHO), adverse events following immunization (AEFI) are any untoward medical event that follows immunization, and that does not necessarily have a causal relationship with the usage of the vaccine. The adverse event may be any unfavorable or unintended sign, abnormal laboratory finding, symptom, or disease. Case definitions must be developed, and a series of systematic activities must be followed to reach a solid conclusion [6]. 
Passive (automatic), active, cohort event monitoring (CEM), and sentinel are the four main surveillance methods. For AEFI, improving existing vaccine safety surveillance systems across the world is crucial. Although passive systems are most widely used to collect AEFIs, adverse events of particular interest (AESI), monitoring through active surveillance should be considered by countries with more robust systems. In order to improve vaccine safety monitoring, all stakeholders should be involved. The system should include data collection and report tools after notification, investigation, analysis, causality assessment, and communication [6].

\section{Data collection}

The European Medicines Agency (EMA) defines a safety signal as "Information on a new or known adverse event that is potentially caused by a medicine, and that warrants further investigation." Passive surveillance systems are wildly used to detect signals of unanticipated events that may be causally related to vaccination. [7]. Passive surveillance systems are inexpensive to set up and operate, but they have considerable limitations. Few reports of AEFIs to passive surveillance systems cannot determine if the event is caused by the vaccine or coincidental as there is a lack of a comparison (unvaccinated) group. There are as many potential biases as possible in reports, including inadequate case definitions, under, over, or incomplete reporting. Passive reports are also prone to misinterpretation by the public, who can mistakenly assume that the AEs reported are caused by the vaccine [6]. In passive surveillance, vaccination-related AEFIs are most likely to be reported if they occur close to the time of vaccination, while events more distant from vaccination may not be reported even if they are causally related. AEFIs that occur with no clear temporal relationship to vaccination is the most challenging to assess. Most surveillance systems for AEFIs are not set up to detect such associations. For example, the increased risk of severe dengue in seronegative vaccines was only discovered in the third year after vaccination. Vaccine risk must be viewed alongside the benefits, and the risk-benefit ratio may vary. Between high and low-middle-income countries, as are the case with intussusception and rotavirus vaccines [8].

In active surveillance, the designated staff visit healthcare facilities, communicate with healthcare providers, review patient records, detect possible cases of AESI, and collect and analyze data using a standard protocol. The advantages of this system are higher sensitivity and can be used for signal detection, determine the rate of an event in a defined population, and determine the relative risk of the event. However, it is resource and time-consuming, lower coverage, and prone to biases unless blinded observers are used [6].

\section{Signal detection and management}

Any signal will pass through different steps before being confirmed, including detection and reporting, validation, conformation, analysis/ prioritization, assessment, and recommendation for action. When investigating events that occur after taking vaccines or medications, signals may emerge from an observed association. Thus, potential signals should always be studied further and should not be taken as evidence of a safety issue [8]. Most AEFIs that are causally linked with vaccination occurs within a few weeks after vaccination (e.g., narcolepsy following pandemic flu vaccination, intussusception following rotavirus vaccination). The plausibility of causal associations is increased by such temporal associations, poor understanding of the disease etiology, and challenges in disease diagnostics affect vaccine safety studies [8].

The Brighton Collaboration, with the Coalition for Epidemic Preparedness Innovations (CEPI) and the WHO, receives feedback from regulatory agencies and other experts to agree on a list of adverse events of particular interest (AES). AESI is a pre-specified medically significant event that can be causally associated with a vaccine product that needs to be carefully monitored and confirmed by further special studies. Identification is made via any active surveillance system: sentinel sites or electronic health records (EHR-based cohort studies). COVID-19 vaccines AESI(s) are designed to find predefined specific events using a set of criteria to see if they are associated with COVID-19 vaccination or not [9].

The AESI list covers all body systems, including immunological, cardiovascular, neurological, musculoskeletal, and dermatological manifestations and associated case definitions and surveillance strategies. Anaphylaxis, vasculitis, myocarditis, generalized convulsions, and meningoencephalitis are among the conditions listed. Comprehensive safety data is required to ensure that the benefit: risk ratio of the vaccine is favorable, allowing policymakers to make informed decisions about implementing a large-scale program among healthy citizens and ensuring that people are confident enough to accept vaccination [9]. AESI will require post-marketing (phase 4) studies, but they are also being evaluated in pre-clinical and early phase trials, particularly the more extensive phase 3 trials. Although the mRNA, adenovirus, and adjuvanted protein nanoparticle constructs all had appropriate safety profiles, the inactivated whole virus vaccine was significantly less local and systemic reactogenic. The reactions were mainly mild to moderate, and they disappear within a few days [9].

\section{Causality assessment \\ Different methods for determining the causal association between adverse reactions and vaccines include Austin Bradford Hill's concepts and subsequent suggested modifications by Fedak. Other methods include causal inference methods, which test causal associations using algorithms and other statistical methods $[8,10,11]$.}

It is very challenging to study the interactions between the vaccine, natural disease, and the AEFI. Interactions between natural disease and the vaccine may be studied for safety in populations with a low burden of natural disease during vaccine development and clinical trials. Many health outcomes differ 
with age, and if a high-incidence adverse reaction occurs shortly after introducing a vaccine, the temporal association can be easily misinterpreted as causal. The observed association (the event occurs after taking the drug) is not the same as the causal association (the event occurs due to the drug). The reason for this misinterpretation is that other factors such as chance, bias, and confusion may all play a role in whether a drug tends to be linked to an event. For instance, in COVID-19, numerous observational studies have looked at the use of experimental treatments and their association with disease recovery. However, there may be various reasons for the patient's recovery, and it is not only due to the use of potential treatments. It is crucial to rule out that any observed associations are due to something else [8].

\section{Risk Management Plans of COVID-19 vaccines}

The post-marketing quality, effectiveness, and safety of COVID19 vaccines approved by the fast-track mechanism must be comparable to their pre-marketing clinical trials. A risk management plan (RMP) in the marketing authorization process is a regulatory requirement. The RMP identifies critical potential risks, substantial identified risks, and missing information documented during the marketing authorization process.

If the RMP suggests post-authorization safety and/or efficacy studies (as is the case for almost all new vaccines and medicines), they must be performed with agreed-upon milestones. Any concern affecting the benefit/risk balance will determine the timing and duration of interim reporting and the completion of the final post authorization study report [6].

For COVID-19 vaccines and medicines, early and regular interim reporting is required. Although RMPs are required for each product, they are not required for all products. A single postauthorization study can include evaluating the safety and/or efficacy of several products if appropriate, specifically if they are going to be given at the same time [6].

The WHO is one of the most important partners to countries in deploying and studying the safety of COVID-19 vaccines besides other national, regional, and international partners. Many member states are part of the WHO International Drug Monitoring Program. The reports related to AEFIs are sent to the VigiBase ${ }^{\circledR}$ (international adverse event database). Uppsala Monitoring Center (UMC), a WHO collaborating center in Sweden, is responsible for maintaining this database [15].

One of the methods of communicating safety data is through the SmPC. SmPC safety sections include contra-indication (4.3), special warning and precautions of use (4.4), interactions with other medicinal products (4.5), fertility, pregnancy, and lactation (4.6), effects on the ability to drive (4.7), undesirable effects (4.8); and overdose (4.9).
All the regulated safety data will be presented in the different safety sections of the clinical part of the SmPC, and all topics/events discussed in section 4.4 are by default substantial risks (but not necessarily identified). The objective of section 4.4 is to provide information on a specific risk when healthcare professionals have to be warned of this risk, or the risk leads to a precaution for use to avoid harm. All events tabulated in section 4.8 are by default-identified risks (but not necessarily essential) [14].

\section{The results of COVID-19 vaccines safety}

The UMC publishes a monthly descriptive report of the COVID19 vaccines AEFIs shared by 144 member states with VigiBase ${ }^{\circledR}$ to help in studying trends of AEFIs. This report comes with a caveat that some of the data presented are not yet clinically thoroughly investigated and need further analysis. In addition, the nature of the shared reports (voluntary) might also have an intrinsic bias that may cause misinterpretation of the observed association with the reported outcome. The $5^{\text {th }}$ report was published on May 26, 2021. The report included information related to each of the currently available vaccines across the world. Table 1 shows the latest available information from VigiBase $^{\circledR}$ as of May 26, 2021, with a total number of 900.943 reports [16].

The rare thrombosis with thrombocytopenia syndrome (TTS) that causes unusual blood clots associated with low numbers of platelets was also reported to VigiBase ${ }^{\circledR}$. However, the mechanism of its occurrence is not fully understood. Both female gender and age were marked as identified risk factors for TTS. Reviewing the available data suggest a causal relationship between TTS and adenoviral vectored vaccine (AstraZeneca and J\&J vaccines) and not with the MRNA COVID19 vaccines $[17,18]$. The risk of TTS with $A Z$ vaccine in the UK was found to be (1 case per 250000 ), and around 1 per 100, 000 in the European Union (EU).

Accordingly, a specific case definition was developed by the Brighton collaboration addressing TTS. The WHO interim guidance on using the AstraZeneca vaccines against COVID-19 has been updated to include additional precautions and types of risk (i.e., TTS Syndrome). Many regulatory authorities besides the WHO continue to believe that the benefits of these vaccines outweigh the risks and will continue the safety monitoring of all vaccines [19].

The opportunities of rapid development and validation of novel technologies coordinated, and collaborative approaches are very evident. However, challenges are multi-dimensional, including but not limited to 1) multiple innovative vaccines are being rolled out simultaneously, with billions of doses being provided to various populations and settings, 2) safety implications in priority target populations and mass immunization campaigns, 3) the need for systems to rapidly detect signals, 4) near real-time data analysis and communication, 5) rapid response to safety signals, 
6) and unique challenges never faced before such as risk of mishandling of information, and vaccination errors.

\section{Conclusions}

The need for the safety monitoring of the COVID-19 vaccine is unprecedented. It is an ongoing process starting from different phases of clinical trials and continued to post-marketing to tackle the emergency used authorized COVID-19 vaccines across the world. Rapid detection, exchange, prioritization, and assessment of safety signals based on available real-world data, surveillance of AEFI and AESI by studying the observed/expected cases are essential. Enhanced collaboration, and availability of resources, tools and methods will add to the lessons learned from previous experiences.

\section{References}

1. Krammer F. SARS-CoV-2 vaccines in development. Nature. 2020 Oct;586(7830):516-27.

2. Flanagan KL, Best E, Crawford NW, Giles M, Koirala A, Macartney K, Russell F, The BW, Wen SC. Progress and Pitfalls in the Quest for Effective SARS-CoV-2 (COVID-19) Vaccines. Frontiers in immunology. 2020 Oct 2;11:2410.Soiza RL, Scicluna C, Thomson EC. Efficacy and safety of COVID-19 vaccines in older people. Age and Ageing. $2021 \mathrm{Mar} ; 50(2): 279-83$.

3. Dal-Ré R, Bekker LG, Gluud C, Holm S, Jha V, Poland GA, Rosendaal FR, Schwarzer-Daum B, Sevene E, Tinto $\mathrm{H}$, Voo TC. Ongoing and future COVID-19 vaccine clinical trials: challenges and opportunities. The Lancet Infectious Diseases. 2021 May 18.

4. Calina D, Docea AO, Petrakis D, Egorov AM, Ishmukhametov AA, Gabibov AG, Shtilman MI, Kostoff R, Carvalho F, Vinceti M, Spandidos DA. Towards effective COVID-19 vaccines: Updates, perspectives, and challenges. International journal of molecular medicine. 2020 Jul 1;46(1):3-16.

5. London School of Hygiene and Tropical Medicine: https://vacIshtm.shinyapps.io/ncov vaccine landscape/. Accessed May 28, 2021.

6. WHO, COVID-19 vaccines: safety surveillance manual. https://worldhealthorganization.odoo.com/modules. Accessed May 29, 2021.

7. European Medicines Agency. Safety signal https://www.ema.europa.eu/en/glossary/safetysignal. Accessed May 28, 2021.

8. Osborne V, Shakir SA. What Is the Difference Between Observed Association and Causal Association, Signals and Evidence? Examples Related to COVID-19. Frontiers in Pharmacology. 2020;11.

9. Safety Platform for Emergency vACcines (SPEAC). https://brightoncollaboration.us/covid-19/. Accessed May 252021.
10. Hill $A B$. The environment and disease: association or causation?. Journal of the Royal Society of Medicine. 2015 Jan;108(1):32-7.

11. Fedak, K. M., Bernal, A., Capshaw, Z. A., and Gross, S. (2015). Applying the Bradford Hill criteria in the 21st century: howdata integration has changed causal inference in molecular epidemiology. Emerg. Themes Epidemiol. 12, 14. doi:10.1186/s12982-015-0037-4

12. S Shakir, S Lane, M Davies. How to Investigate a Serious Adverse Event Reported During a Clinical Trial for a COVID-19 Vaccine. Drug Saf, volume 44, issue 1. Posted 2021.

13. Dhanda S, Osborne V, Lynn E, Shakir S. Postmarketing studies: can they provide a safety net for COVID-19 vaccines in the UK?. BMJ evidence-based medicine. 2020 Oct 21.

14. European Commission. A guideline on summary of product

characteristicsSmPC).https://ec.europa.eu/health/sit es/default/files/files/eudralex/vol-

$2 / c / s m p c$ guideline rev2 en.pdf. Accessed May 24 2021.

15. Uppsala Monitoring Centre: UMC, https://www.whoumc.org. Accessed 24 May 2021.

16. Uppsala Monitoring Center. The Vigibase. Accessed May 262021.

17. WHO. Statement of the COVID-19 subcommittee of the WHO Global Advisory Committee on Vaccine Safety (GACVS) on safety signals related to the Johnson \& Johnson/Janssen COVID-19 vaccine. https://www.who.int/news/item/19-05-2021statement-gacvs-safety-johnson-johnson-janssencovid-19-vaccine. Accessed May 282021.

18. WHO. Global Advisory Committee on Vaccine Safety (GACVS) review of latest evidence of rare adverse blood coagulation events with AstraZeneca COVID-19 Vaccine (Vaxzevria and Covishield) - Apr 16 2021: https://www.who.int/news/item/16-04-2021-globaladvisory-committee-on-vaccine-safety-(gacvs)review-of-latest-evidence-of-rare-adverse-bloodcoagulation-events-with-AstraZeneca-covid-19vaccine-(vaxzevria-and-covishield).

19. WHO. Statement of the Strategic Advisory Group of Experts (SAGE) on Immunization: Continued review of emerging evidence on AstraZeneca COVID-19 Vaccines. https://www.who.int/news/item/22-042021-statement-of-the-strategic-advisory-group-ofexperts-(sage)-on-immunization-continued-review-ofemerging-evidence-on-AstraZeneca-covid-19vaccines. Accessed 28 May 2021. 
Table 1: Characteristics of the reported adverse events following immunization (AEFI) cases to VigiBase ${ }^{\bullet(16)}$

\begin{tabular}{|c|c|c|c|}
\hline \multicolumn{2}{|l|}{ Characteristics } & \multirow{2}{*}{$\begin{array}{l}\text { Number (n) } \\
900,943\end{array}$} & \multirow{2}{*}{$\begin{array}{l}\text { Percentage (\%) } \\
100 \%\end{array}$} \\
\hline \multirow{10}{*}{ COVID-19 vaccine } & All vaccines & & \\
\hline & COVID-19 vaccine NRVV Ad (ChAdOx1 nCoV-19) AstraZeneca & 374,647 & $41.6 \%$ \\
\hline & Tozinameran (Comirnaty, Pfizer BioNTech) & 327,908 & $36.4 \%$ \\
\hline & COVID-19 vaccine mRNA (mRNA 1273) Moderna & 129,046 & $14.3 \%$ \\
\hline & COVID-19 vaccine NRVV Ad26 (JNJ 78436735) Janssen & 37,216 & $4.1 \%$ \\
\hline & COVID-19 vaccine inact (Vero) CZ02 CoronaVac, Vero Cell, Sinovac & 15,847 & $1.8 \%$ \\
\hline & COVID-19 vaccine inact (Vero) HB02 Vero Cell, Sinopharm, Beijing & 7,708 & $0.9 \%$ \\
\hline & Covid-19 vaccine & 5,201 & $0.6 \%$ \\
\hline & Covid-19 vaccine inact (Vero) & 1,744 & $0.2 \%$ \\
\hline & COVID-19 vaccine NRVV Ad26 (Gam-Covid-Vac) (Sputnik V) & 1,183 & $0.1 \%$ \\
\hline \multirow[t]{5}{*}{ Age of patient } & $<18$ years & 4,374 & $0.1 \%$ \\
\hline & $18-44$ years & 344,786 & $38.3 \%$ \\
\hline & $45-64$ years & 320,355 & $35.6 \%$ \\
\hline & $65-74$ years & 97,842 & $10.9 \%$ \\
\hline & $\geq 75$ years & 72,001 & $8.0 \%$ \\
\hline \multirow[t]{3}{*}{ Gender } & Female & 652,019 & $72.4 \%$ \\
\hline & Male & 232,851 & $25.9 \%$ \\
\hline & unknown & 15,825 & $1.8 \%$ \\
\hline \multirow{10}{*}{$\begin{array}{l}\text { Top Reported } \\
\text { preferred terms } \\
\text { (MedDRA) }\end{array}$} & PT: Headache & 289,064 & $32.1 \%$ \\
\hline & PT: Pyrexia & 236,027 & $26.2 \%$ \\
\hline & PT: Fatigue & 185,095 & $20.6 \%$ \\
\hline & PT: Chills & 172,562 & $19.2 \%$ \\
\hline & PT: Myalgia & 148,988 & $16.5 \%$ \\
\hline & PT: Nausea & 124,454 & $13.8 \%$ \\
\hline & PT: Arthralgia & 98,604 & $10.9 \%$ \\
\hline & PT: Malaise & 87,258 & $9.7 \%$ \\
\hline & PT: Injection site pain & 80,064 & $8.9 \%$ \\
\hline & PT: Dizziness & 73,599 & $8.2 \%$ \\
\hline \multirow[t]{3}{*}{ Seriousness } & Yes & 231,592 & $25.7 \%$ \\
\hline & No & 667,052 & $74.1 \%$ \\
\hline & unknown & 2,051 & $0.2 \%$ \\
\hline \multirow{5}{*}{$\begin{array}{l}\text { Seriousness } \\
\text { Criteria }\end{array}$} & Death & 10,842 & $1.2 \%$ \\
\hline & Life-threatening & 12,502 & $1.4 \%$ \\
\hline & Caused/prolonged hospitalization & 37,266 & $4.1 \%$ \\
\hline & Disabling/incapacitating & 24,142 & $2.7 \%$ \\
\hline & Congenital anomaly/birth defect & 126 & $0.0 \%$ \\
\hline
\end{tabular}

